\title{
Physics of the GRB 030328 afterglow and its environment ${ }^{\star} \star \star \star$
}

\author{
E. Maiorano ${ }^{1,2,3}$, N. Masetti ${ }^{1}$, E. Palazzi ${ }^{1}$, S. Savaglio ${ }^{4}$, E. Rol ${ }^{5}$, P. M. Vreeswijk ${ }^{6}$, E. Pian ${ }^{1,2}$, P. A. Price ${ }^{7,8}$,
} B. A. Peterson ${ }^{8}$, M. Jelínek ${ }^{9}$, L. Amati ${ }^{1}$, M. I. Andersen ${ }^{10}$, A. J. Castro-Tirado ${ }^{9}$, J. M. Castro Cerón ${ }^{11}$, A. de Ugarte Postigo ${ }^{9}$, F. Frontera ${ }^{1,12}$, A. S. Fruchter ${ }^{13}$, J. P. U. Fynbo ${ }^{11}$, J. Gorosabel ${ }^{9}$, A. A. Henden ${ }^{14}$, J. Hjorth ${ }^{11}$, B. L. Jensen ${ }^{11}$, S. Klose ${ }^{15}$, C. Kouveliotou ${ }^{16}$, G. Masi ${ }^{17}$, P. Møller ${ }^{18}$, L. Nicastro ${ }^{1}$, E. O. Ofek ${ }^{19}$, S. B. Pandey ${ }^{9,20}$, J. Rhoads ${ }^{13}$, N. R. Tanvir ${ }^{21}$, R. A. M. J. Wijers ${ }^{22}$, and E. P. J. van den $\mathrm{Heuvel}^{22}$

${ }^{1}$ INAF - Istituto di Astrofisica Spaziale e Fisica Cosmica di Bologna, via Gobetti 101, 40129 Bologna, Italy e-mail: maiorano@iasfbo.inaf.it

2 INAF - Osservatorio Astronomico di Trieste, via G.B. Tiepolo 11, 34131 Trieste, Italy

3 Dipartimento di Astronomia, Università di Bologna, via Ranzani 1, 40126 Bologna, Italy

4 The Johns Hopkins University, 3400 North Charles Street, Baltimore, MD 21218, USA

5 Department of Physics and Astronomy, University of Leicester, University Road, Leicester, LE1 7RH, UK

6 European Southern Observatory, Casilla 19001, Santiago 19, Chile

7 Institute for Astronomy, University of Hawaii, 2680 Woodlawn Drive, Honolulu, HI 96822, USA

8 Research School of Astronomy and Astrophysics, Australian National University, via Cotter Road, Weston, ACT 2611, Australia

9 Instituto de Astrofísica de Andalucía (IAA-CSIC), PO Box 03004, 18080 Granada, Spain

${ }_{10}$ Astrophysikalisches Institut, 14482 Potsdam, Germany

11 Dark Cosmology Centre, Niels Bohr Institute, University of Copenhagen, Juliane Maries Vej 30, 2100 Copenhagen Ø, Denmark

12 Dipartimento di Fisica, Università di Ferrara, via Saragat 1, 44100 Ferrara, Italy

3 Space Telescope Science Institute, 3700 San Martin Drive, Baltimore, MD 21218, USA

14 Universities Space Research Association / US Naval Observatory, PO Box 1149, Flagstaff, AZ 86002, USA

15 Thüringer Landessternwarte Tautenburg, 07778 Tautenburg, Germany

16 NASA MSFC, SD-50, Huntsville, AL 35812, USA

17 Dipartimento di Fisica, Università di Roma "Tor Vergata”, via della Ricerca Scientifica 1, 00133 Rome, Italy

18 European Southern Observatory, Karl Schwarzschild-Strasse 2, 85748 Garching, Germany

19 School of Physics and Astronomy and the Wise Observatory, University of Tel-Aviv, Tel-Aviv 69978, Israel

20 ARIES Observatory, Manora Peak, Naini Tal, 263129 Uttaranchal, India

21 Department of Physical Sciences, University of Hertfordshire, College Lane, Hatfield, Herts AL10 9AB, UK

22 Institute of Astronomy "Anton Pannekoek", University of Amsterdam, Kruislaan 403, 1098 SJ Amsterdam, The Netherlands

Received 20 December 2005 / Accepted 19 March 2006

\section{ABSTRACT}

Aims. To investigate the physical nature of the afterglow emission. We report on the photometric, spectroscopic and polarimetric observations of the optical afterglow of Gamma-Ray Burst (GRB) 030328 detected by HETE-2.

Methods. Photometric, spectroscopic and polarimetric monitoring of the optical afterglow.

Results. Photometry, collected at 7 different telescopes, shows that a smoothly broken powerlaw decay, with indices $\alpha_{1}=0.76 \pm$ $0.03, \alpha_{2}=1.50 \pm 0.07$ and a break at $t_{\mathrm{b}}=0.48 \pm 0.03$ days after the GRB, provides the best fit of the optical afterglow decline. This shape is interpreted as due to collimated emission, for which we determine a jet opening angle $\theta_{\text {jet }} \sim 3^{\circ} .2$. An achromatic bump starting around $\sim 0.2 \mathrm{~d}$ after the GRB is possibly marginally detected in the optical light curves. Optical spectroscopy shows the presence of two rest-frame ultraviolet metal absorption systems at $z=1.5216 \pm 0.0006$ and at $z=1.295 \pm 0.001$, the former likely associated with the GRB host galaxy. Analysis of the absorption lines at $z=1.5216$ suggests that the host of this GRB may be a Damped Lyman- $\alpha$ Absorber. The optical $V$-band afterglow appears polarized, with $P=(2.4 \pm 0.6) \%$ and $\theta=170^{\circ} \pm 7^{\circ}$, suggesting an asymmetric blastwave expansion. An X-ray-to-optical spectral flux distribution of the GRB 030328 afterglow was obtained at 0.78 days after the GRB and fitted using a broken powerlaw, with an optical spectral slope $\beta_{\text {opt }}=0.47 \pm 0.15$, and an X-ray slope $\beta_{\mathrm{X}}=1.0 \pm 0.2$.

Conclusions. The discussion of the results in the context of the "fireball model" shows that the preferred scenario for this afterglow is collimated structured jet with fixed opening angle in a homogeneous medium.

Key words. gamma rays: bursts - radiation mechanisms: non-thermal - line: identification - cosmology: observations

^ Based on observations collected with: the Nordic Optical Telescope (NOT) operating on the island of La Palma in the Spanish Observatorio del Roque de los Muchachos of the Instituto de Astrofísica de Canarias (Spain), the 1.54 Danish telescope and the $2.2 \mathrm{~m} \mathrm{ESO/MPG} \mathrm{telescope}$ (the latter under ESO Program ID: 70.D-0523) both operating at ESOLa Silla (Chile), the VLT plus FORS1 (ESO Program IDs: 70.D-0523 and 70.D-0531) at ESO-Paranal (Chile), the 40-inch of Siding Spring
Observatory (Australia), the 1m Sampurnanand telescope at the ARIES observatory (India), and the $1 \mathrm{~m}$ NOFS telescope of USNO in Flagstaff (USA).

$\star \star$ Table 1 is only available in electronic form at

http://www . edpsciences.org 


\section{Introduction}

Long Gamma-Ray Bursts (GRBs) are rapid and powerful highenergy events lasting more than $\sim 2 \mathrm{~s}$ and up to $\sim 1000 \mathrm{~s}$ (e.g., Kouveliotou et al. 1993). The detection of their longerwavelength counterparts (Costa et al. 1997; van Paradijs et al. 1997; Guarnieri et al. 1997; Frail et al. 1997), has made it clear that multiwavelength studies of these phenomena are critical in order to constrain the parameters of the emission models and of the circumburst medium (see e.g. Galama et al. 1998a; Palazzi et al. 1998; Masetti et al. 1999).

The discovery that the optical emission of GRB afterglows is polarized at the level of a few percent (Covino et al. 1999; Wijers et al. 1999) points to the relevance of asymmetries in the afterglow emission (Rol et al. 2000, 2003a; Greiner et al. 2003a) possibly induced by the presence of a collimated jet (Sari 1999; Ghisellini \& Lazzati 1999). However, there have been few polarimetric measurements of GRB optical afterglows so far, due to the rapid fading of these sources and to the relatively low level of the polarized signal, requiring timely observations with large telescopes.

Due to their high intrinsic brightness in the early emission phases, long GRBs are often used as a probe with which to sample the metal content of their host galaxies (e.g., Savaglio et al. 2003) and thus are a powerful tool with which to map the metallicity evolution in the Universe at very high $(z>1)$ redshifts. This is an important parameter with which to characterize the metallicity content of the environments where the massive progenitors of GRBs originate (Galama et al. 1998b; Iwamoto et al. 1998; Hjorth et al. 2003; Stanek et al. 2003; Matheson et al. 2003; Malesani et al. 2004; Woosley \& Heger 2006).

GRB 030328 was a long $\left(T_{90} \sim 100 \mathrm{~s}\right.$ at $\left.30-400 \mathrm{keV}\right)$, bright (fluence: $S_{\gamma}=3.0 \times 10^{-5} \mathrm{erg} \mathrm{cm}{ }^{-2}$; peak flux: $F_{\gamma}=7.3 \times$ $10^{-7} \mathrm{erg} \mathrm{cm}^{-2} \mathrm{~s}^{-1}$ ) GRB detected on 2003 Mar. 28.4729 UT, by the FREGATE, WXM, and SXC instruments onboard HETE-2, and rapidly localized with sub-arcminute accuracy (Villasenor et al. 2003). About $\sim 1 \mathrm{~h}$ after the GRB, its optical afterglow was detected by the 40-inch Siding Spring Observatory (SSO) telescope in Australia at coordinates (J2000) RA $=12^{\mathrm{h}} 10^{\mathrm{m}} 48^{\mathrm{s}} .4$, Dec $=-09^{\circ} 20^{\prime} 51^{\prime \prime} .3$ with a magnitude $R \sim 18$ (Peterson $\&$ Price 2003; Price \& Peterson 2003). Martini et al. (2003a) measured the redshift of this Optical Transient (OT), $z=1.52$, based on the identification of $\mathrm{Mg}$ II, Fe II and Al II absorption. This redshift was confirmed by Rol et al. (2003b), who also reported the possible detection of a foreground absorption system at redshift $z=1.29$, and by Fugazza et al. (2003a). Multicolor imaging and analysis of the host galaxy was reported by Gorosabel et al. (2005). Chandra observations, starting about $15 \mathrm{~h}$ after the GRB, revealed a new, fading $X$-ray source at a position consistent with that of the optical transient, identified with the X-ray afterglow (Butler et al. 2005). Its temporal decrease followed a powerlaw with index $\alpha_{\mathrm{X}}=1.5 \pm 0.1$, and its $0.5-5 \mathrm{keV}$ spectrum was well fitted by an absorbed power-law of photon index $\Gamma_{X}=2.0 \pm$ 0.2 . The $N_{\mathrm{H}}$ column density derived from the spectral fit, $(5 \pm$ 3) $\times 10^{20} \mathrm{~cm}^{-2}$, is consistent with the Galactic foreground value (Dickey \& Lockman 1990).

We report here on the study of the optical afterglow emission of GRB 030328. The paper is organized as follows: Sect. 2 describes the observations and data reduction; the photometric, spectroscopic and polarimetric results together with the multiwavelength spectrum are reported in Sect. 3; discussion and conclusions are presented in Sect. 4.
Most of the data presented in this paper were acquired, with programs led by the GRACE ${ }^{1}$ collaboration, at the Nordic Optical Telescope (NOT) in the Canary Islands (Spain) and at the European Southern Observatory (ESO) telescopes of Cerro Paranal and La Silla (Chile), starting $\sim 11 \mathrm{~h}$ after the high-energy prompt event. Observations acquired with the 40-inch SSO telescope, the $1 \mathrm{~m}$ Sampurnanand telescope at the Aryabhatta Research Institute of Observational Sciences (ARIES) in Naini Tal (India) and with the $1 \mathrm{~m}$ US Naval Observatory Flagstaff Station (NOFS) telescope (Arizona, USA) are also included in this paper, along with the analysis of public VLT optical polarimetry data.

We remark that, although GRB 030328 was a bright event, it was not monitored intensively after the first hours, because it occurred the day before GRB 030329, the extremely bright, low redshift ( $z=0.168$; Greiner et al. 2003b) event triggered by HETE-2 (Vanderspek et al. 2003) and unambiguously associated with a supernova (Stanek et al. 2003; Hjorth et al. 2003), which caught all the observers' attention over subsequent weeks.

Throughout this paper we will assume a cosmology with $H_{0}=65 \mathrm{~km} \mathrm{~s}^{-1} \mathrm{Mpc}^{-1} ; \Omega_{\Lambda}=0.7 ; \Omega_{\mathrm{m}}=0.3$; also, when not otherwise indicated, errors and upper limits will be given at $1 \sigma$ and $3 \sigma$ confidence levels, respectively.

\section{Observations and data reduction}

\subsection{Photometry}

Imaging in the Johnson-Cousins UBVRI and Gunn $i$ filters was performed between March 28 and March 31 at the $2.5 \mathrm{~m}$ NOT telescope in La Palma with the ALFOSC instrument. This is equipped with a $2048 \times 2048$ pixel Loral CCD, giving a field of view of $6^{\prime} 4 \times 6.4$ and an image scale of $0.188 \mathrm{pix}^{-1}$.

Optical BVRI data were also obtained on 2003 Mar. 29 using the $2.2 \mathrm{~m}$ ESO/MPG and $1.54 \mathrm{~m}$ Danish telescopes at La Silla. The Wide Field Imager (WFI), permanently mounted on the $2.2 \mathrm{~m} \mathrm{ESO/MPG}$ telescope, is a focal reducer-type camera, compraising a mosaic of $82048 \times 4096$ pixel CCDs. The WFI provides a field of view of $34^{\prime} \times 33^{\prime}$ and an image scale of $0^{\prime} .238 \mathrm{pix}^{-1}$. The DFOSC is equipped with a $2048 \times$ 2048 pixels CCD covering a 13:7 × 13!7 field, with a spatial resolution of $0.39 \mathrm{pix}^{-1}$. On the same night, $V$-band data were acquired with VLT-Antu plus FORS1 at Cerro Paranal. FORS1 is equipped with a $2048 \times 2048$ pixel Tektronix CCD which covers a $6.8 \times 6.8$ field in the standard resolution imaging mode with a scale of 0.2 pix $^{-1}$.

UBVRI frames were also acquired with the $1 \mathrm{~m}$ NOFS telescope. This telescope carries a $1024 \times 1024$ pixel Tektronix CCD which can image a field of $12^{\prime} \times 12^{\prime}$ with a scale of 0.68 pix $^{-1}$.

Earlier observations in the $B$ and $R$ filters were obtained with the 40-inch SSO telescope and with the $1 \mathrm{~m}$ ARIES telescope. The 40-inch telescope was equipped with a WFI, consisting of 8 $2048 \times 4096$ pixel CCDs, which cover a field of view of $52^{\prime} \times$ $52^{\prime}$ with an image scale of $0.38 \mathrm{pix}^{-1}$. The $1 \mathrm{~m}$ ARIES telescope carries a $2048 \times 2048$ pixel CCD, with a $13^{\prime} \times 13^{\prime}$ field of view; in its standard $2 \times 2$ binning mode it has a spatial resolution of $0.76 \mathrm{pix}^{-1}$.

Optical frames were bias-subtracted and flat-fielded with the standard cleaning procedure. We chose standard Point Spread Function (PSF) fitting to perform photometry on the images, and

\footnotetext{
1 GRB Afterglow Collaboration at ESO: see the web page http://www.gammaraybursts . org/grace/
} 
used the DAOPHOT II image data analysis package PSF-fitting algorithm (Stetson 1987) running within MIDAS ${ }^{2}$. A twodimensional Gaussian profile with two free parameters (the half width at half maxima along the $x$ and $y$ coordinates of each frame) was modeled on at least 5 unsaturated bright stars in each image.

The UBVRI zero-point calibration was performed using the photometry by Henden (2003). We then selected stars of varoius brightness in the GRB 030328 field and used them to determine the $U B V R I$ magnitudes of the OT. No color-term correction was computed as we chose stars with color indices as close as possible to those of the OT. The errors associated with the measurements reported in Table 1 account for both statistical uncertainties obtained with the standard PSF-fitting procedure and systematic errors of the magnitude calibration. The single Gunn $i$ image acquired with the NOT was calibrated using the $I$-band secondary standards, given that the widths, the reference wavelengths and the flux density normalizations of the two filters are very similar (Fukugita et al. 1995). However, in order to account for small differences between the filters, we added in quadrature a $3 \%$ uncertainty to the magnitude error obtained from the Gunn $i$ observation.

All data reported in this paper are listed in Table 1 and are shown in Fig. 2, where we also report those available in the GCN Circulars' archive ${ }^{3}$, rescaled to the photometry of Henden (2003) when needed. For the cases in which no error was reported, a 0.3 mag uncertainty was assumed. We did not include the single $R$-band data point of Rumyantsev et al. (2003) because it deviates by about $4 \sigma$ from the more precise and nearlysimultaneous data secured at the $2.5 \mathrm{~m}$ NOT.

The results presented here and obtained from the 40-inch SSO telescope data supersede the preliminary ones of Peterson $\&$ Price (2003) and Price \& Peterson (2003).

The optical data of Fig. 2 were corrected for the Galactic foreground reddening assuming $E(B-V)=0.047 \mathrm{mag}$ (Schlegel et al. 1998): by applying the relation of Cardelli et al. (1989), we derived $A_{B}=0.19 \mathrm{mag}, A_{V}=0.15 \mathrm{mag}, A_{R}=0.12 \mathrm{mag}$ and $A_{I}=0.09$ mag. Next, they were converted into flux densities assuming the normalizations of Fukugita et al. (1995). The host galaxy emission in the $B V R I$ bands was computed from the data of Gorosabel et al. (2005) and subtracted from our optical data set.

\subsection{VLT spectroscopy}

As reported in Table 1, a series of six 10-min spectra was obtained starting on 2003 Mar. 29.060 UT (0.59 d after the GRB) at ESO-Paranal using VLT-Antu plus FORS1 under fairly good weather conditions, with seeing 0 '. $^{\prime}$ and airmass $1.18-1.41$. The $300 \mathrm{~V}$ Grism was used with a nominal spectral coverage of 3600-8000 $\AA$. The slit width was $1^{\prime \prime}$ and the optical spectral dispersion was $2.7 \AA /$ pixel $(\lambda / \Delta \lambda=440)$.

All spectra have been reduced in the $\operatorname{IRAF}^{4}$ environment with the kpnoslit package. After overscan subtraction and flat-fielding, cosmic rays were removed from the images using the L.A. Cosmic routine written by van Dokkum (2001). The spectra were then optimally extracted for each 2D image separately, with an extraction radius of about $2^{\prime \prime}$. The dispersion

${ }^{2}$ MIDAS (Munich Image Data Analysis System) is developed, distributed and maintained by ESO and is available at http://www.eso.org/projects/esomidas/

3 http://gcn.gsfc.nasa.gov/gcn/gcn3_archive.html

4 Available at http://iraf.noao.edu correction was applied, again to each spectrum separately, using an HeNeAr lamp spectrum that was taken in the morning after the science observations. The typical formal error in the wavelength calibration fit was $0.2 \AA$. The separate dispersioncorrected spectra were averaged, and the corresponding Poisson error spectra, calculated by the apall task, were quadratically averaged.

The flux calibration was performed using observations of the standard LTT 7379 (Hamuy et al. 1992, 1994) carried out during the same night as those of the GRB OT spectrum.

We applied a slit-loss correction by fitting a Gaussian profile along the spatial direction of the averaged $2 \mathrm{D}$ spectra, every 4 pixels across the entire dispersion axis (i.e. summing 4 columns before performing the fit). The resulting Gaussian full width at half maximum (FWHM) was then compared to the slit width in order to obtain the slit loss (i.e. the fraction of the surface underneath the Gaussian fit that is outside the slit width) along the dispersion axis. The slit loss profile was then fitted with a polynomial to correct the spectra. Note that this does not correct for any colour-dependent slit losses, but FORS1 has a linear atmospheric dispersion compensator (LADC) in the light path which minimizes any colour-differential slit losses up to a zenith distance of $45^{\circ}$.

\subsection{VLT polarimetry}

Linear polarimetry $V$-band observations (see Table 1 for the observation $\log$ ) were acquired starting on 2003 Mar. 29.130 UT at VLT-Antu plus FORS1 under an average seeing of $\sim 1^{\prime \prime}$. Data were obtained, between 0.66 and $0.88 \mathrm{~d}$ after the GRB, using a Wollaston prism and a half-wavelength, phase-retarder plate. The Wollaston prism separates the incident light into ordinary and extraordinary components, while the phase-retarder plate determines which of the Stokes parameters is measured (U or Q). For each image, a mask producing $22^{\prime \prime}$-wide parallel strips was used to avoid so as overlap of the two components. The observation therefore consisted of four exposures centered at the position of the OT, with the phase-retarder plate at $0^{\circ}, 22^{\circ} .5,45^{\circ}$ and $67^{\circ} .5$. Each angle was imaged 5 times cyclically with an exposure time of $780 \mathrm{~s}$, for a total exposure time of $15600 \mathrm{~s}$ over the 4 angles. Image reduction and analysis was performed as described in Sect. 2.1 for the optical photometry.

In order to evaluate the Stokes $U$ and $Q$ parameters of the OT emission, and thus its polarization percentage $P$ and the position angle of the electric field vector $\theta$, we applied the method described by Di Serego Alighieri (1997). Here $P$ and $\theta$ are obtained by using the relation $S(\phi)=P \cos 2(\theta-\phi)$, in which $S$ depends on the ratio between the fluxes of the ordinary and extraordinary components of the incident beam, and $\phi$ corresponds to the prism rotation angle. The values of $P$ and $\theta$ are calculated by fitting the above relation to the measurements obtained in correspondence with each rotation angle. Moreover, using this formalism, one obtains the two Stokes parameters $Q$ and $U$ from the values of $S\left(0^{\circ}\right)$ and $S\left(45^{\circ}\right)$, respectively.

No significant variation among the values of the $U$ and $Q$ parameters of individual field stars was noted, therefore we computed the average values of these quantities and subtracted them from the corresponding parameters of the OT to remove the instrumental and (local) interstellar polarization. We also checked that the polarization of the selected field stars did not systematically vary across the CCD or with the star magnitudes. 


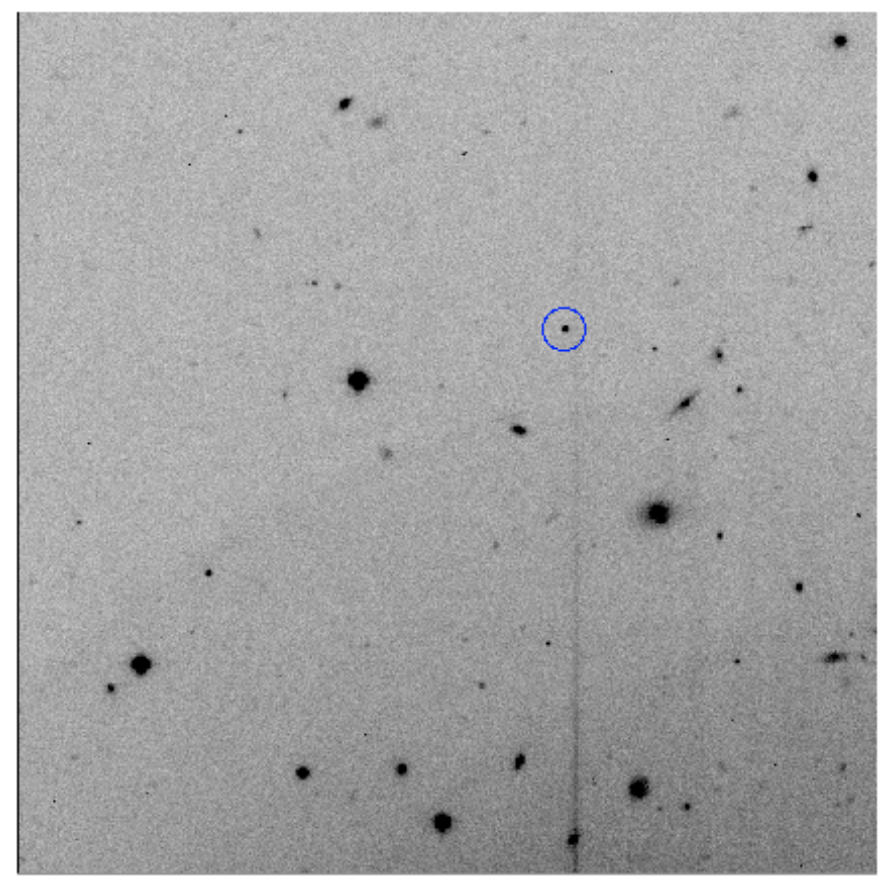

Fig. 1. $R$-band image of the field of the GRB 030328 acquired at ESOLa Silla with the $2.2 \mathrm{~m} \mathrm{ESO/MPG} \mathrm{plus} \mathrm{WFI} \mathrm{on} 2003$ Mar. 29.273 UT. North is at top, East is to the left. The field size is about $2^{\prime} \times 2^{\prime}$. The OT is clearly detected and indicated by the circle.

\subsection{Chandra X-ray data}

In order to determine a precise astrometric position for the X-ray afterglow of GRB 030328, we retrieved the public Chandra/ACIS-S observation ${ }^{5}$ of this source (see Butler et al. 2005 for details). The packages $\mathrm{CIAO}^{6}$ v3.2.2 and $\mathrm{CALDB}^{7}$ v3.1.0 were used for the data reduction. The aspect-solution $90 \%$ confidence level error radius of 0.6 was assumed for the positions of the X-ray sources determined from this observation.

\section{Results}

\subsection{Optical and X-ray astrometry}

The OT (with $R=21.17 \mathrm{mag}$ at $0.80 \mathrm{~d}$ after the GRB; see Table 1) was clearly detected in the WFI $R$-band image (see Fig. 1). An astrometric solution based on 50 USNO-A2.0 8 reference stars in the WFI $R$ image, taken on 2003 Mar. 29.273 UT, yields for the OT the coordinates $(\mathrm{J} 2000) \mathrm{RA}=12^{\mathrm{h}} 10^{\mathrm{m}} 48^{\mathrm{s}} .37$, Dec $=-09^{\circ} 20^{\prime} 51^{\prime \prime}$.39. The internal error of the optical position is 0.238 , which has to be added to the systematic error of the USNO catalogue ( 0.25 according to Assafin et al. 2001 and Deutsch 1999). The final astrometric error is thus 0.'35.

The position of the X-ray source corresponding to the GRB 030328 afterglow in the Chandra data was obtained using the celldetect command. Only a single bright source is detected at the center of the ACIS-S field of view. This does not allow us to tie the internal Chandra astrometry to that of

\footnotetext{
5 Available at http://heasarc.gsfc.nasa.gov

6 Available at http://cxc.harvard.edu/ciao/

7 Available at http://cxc . harvard.edu/caldb/

8 The USNO-A2.0 catalogue is available at http: //archive.eso.org/skycat/servers/usnoa
}

optical and/or near-infrared catalogues in order to improve the positional uncertainty afforded by the ACIS-S X-ray data.

The Chandra/ACIS-S astrometry we obtained for the X-ray afterglow of GRB 030328 is the following (J2000): RA = $12^{\mathrm{h}} 10^{\mathrm{m}} 48^{\mathrm{s}} .384$, Dec $=-09^{\circ} 20^{\prime} 51^{\prime \prime} .71$, with an error of $0 .^{\prime} 6$ on both coordinates. This position lies 0!38 from, and is therefore fully consistent with, that reported above for the GRB 030328 OT.

\subsection{Optical light curves}

In Fig. 2 we plot our photometric measurements together with those reported in the GCN circulars. As this figure shows, the best-sampled curve is the $R$-band, so we will first consider this for the light curve decay fitting.

First we modeled the $R$-band light curve using a single powerlaw with index $\alpha=0.94 \pm 0.01\left(\chi^{2} /\right.$ d.o.f. $=145.8 / 81$, where d.o.f. means "degrees of freedom"), but this model did not satisfactorily describe the data, with the points corresponding to the early $(<0.3 \mathrm{~d}$ after the GRB) and late $(>1 \mathrm{~d}$ after the GRB) observations lying systematically below the best-fit curve. The fit improves significantly by using a smoothly broken powerlaw (see Beuermann et al. 1999). The best fit (with $\chi^{2} /$ d.o.f. $=$ 0.4/79), plotted in Fig. 2, is provided by the temporal indices $\alpha_{1}=0.76 \pm 0.03$ and $\alpha_{2}=1.50 \pm 0.07$ before and after a break occurring at $t_{b}=0.48 \pm 0.03 \mathrm{~d}$ from the GRB trigger, and with $s=4.0 \pm 1.5$ the parameter describing the slope change rapidity.

The above best fit also describes the second well-sampled band, i.e., the $B$ one. Concerning the other optical bands, the number of available data points is not enough to allow a meaningful fitting; however, the best-fit obtained for the $R$-band light curve is again fully consistent with the decay trend in the $V$ and $I$ bands (see Fig. 2). This means that the decay of the OT can be considered as achromatic within the uncertainties of the $R$-band light curve best-fit parameters.

We also note that there is a marginal presence of a deviation from the best-fit curve, in the form of an increase in brightness, starting $\sim 0.2 \mathrm{~d}$ after the trigger and lasting $\sim 0.15 \mathrm{~d}$. This variation is, moreover, apparent in all optical bands and is consistent with being achromatic within our uncertainties.

The optical colors of the OT of GRB 030328 fall in the loci populated by GRB afterglows in the color-color diagrams as illustrated by Šimon et al. (2001).

\subsection{Spectroscopy}

Figure 3 shows the spectrum of the GRB 030328 OT. As already remarked in Sect. 2.2, the resolving power is roughly 440 at $5900 \AA$, corresponding to about $700 \mathrm{~km} \mathrm{~s}^{-1}$, or $13.4 \AA$. Table 2 lists the significant $(3 \sigma)$ lines that we detect, their identification, and rest-frame equivalent width $(\mathrm{EW}) \mathrm{W}_{r}$. Most of the significant features can be identified with metal absorption lines in a system at a redshift of $z=1.5216 \pm 0.0006$. These lines are likely associated with the circumburst gas or interstellar medium (ISM) in the GRB host galaxy. A lower redshift absorption system at $z=1.295 \pm 0.001$ is also found: for it, only two lines can be identified (Fe II $\lambda 2600$ and the unresolved Mg II $\lambda \lambda 2796$, 2803 doublet). Its detection indicates the presence of a foreground absorber.

We estimate metal column densities using the observed EW (Table 2), as described in Savaglio \& Fall (2004). Stringent constraints cannot be derived because the resolution of the spectrum does not allow the detection of the many weak absorption lines. 


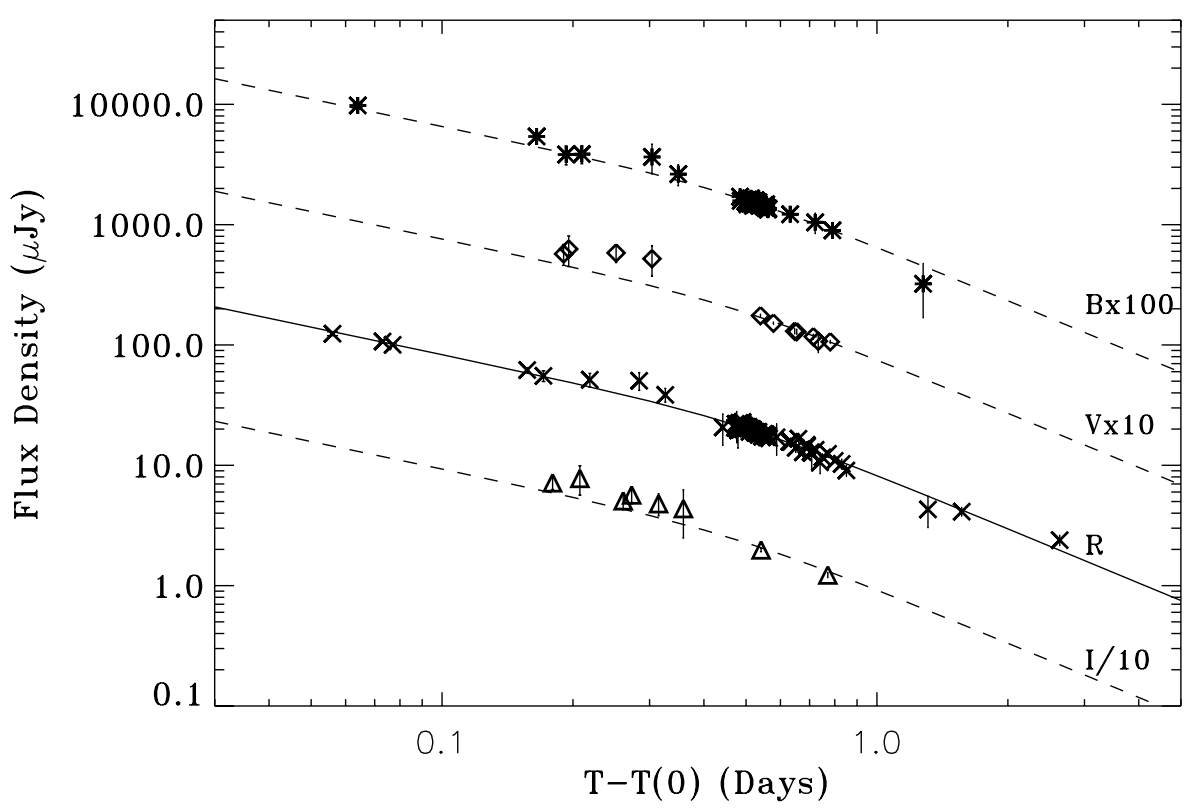

Fig. 2. $B V R I$ light curves of the GRB 030328 afterglow. Upper limits were not plotted. For clarity, $B, V$ and $I$ light curves are shifted by the factors indicated in the figure. Here, $T(0)$ corresponds to the GRB 030328 trigger time (2003 Mar. 28.4729 UT). The data points reported here are from this paper and from Bartolini et al. (2003), Burenin et al. (2003), Fugazza et al. (2003b), Gal-Yam et al. (2003), Garnavich et al. (2003), Ibrahimov et al. (2003) and Martini et al. (2003b). For these measurements, when no error was reported, a 0.3 mag uncertainty was assumed. Data are corrected for the underlying host galaxy contribution and for Galactic absorption. The solid line represents the best-fit of the $R$-band light curve using a broken powerlaw with indices $\alpha_{1}=0.76, \alpha_{2}=1.50$, and break time $t_{\mathrm{b}}=0.48 \mathrm{~d}$ after the GRB. This same model, with unchanged model parameters except for the normalization, is overplotted as a dashed curve onto the $B V I$ data points and describes well the decay behaviour of these light curves.

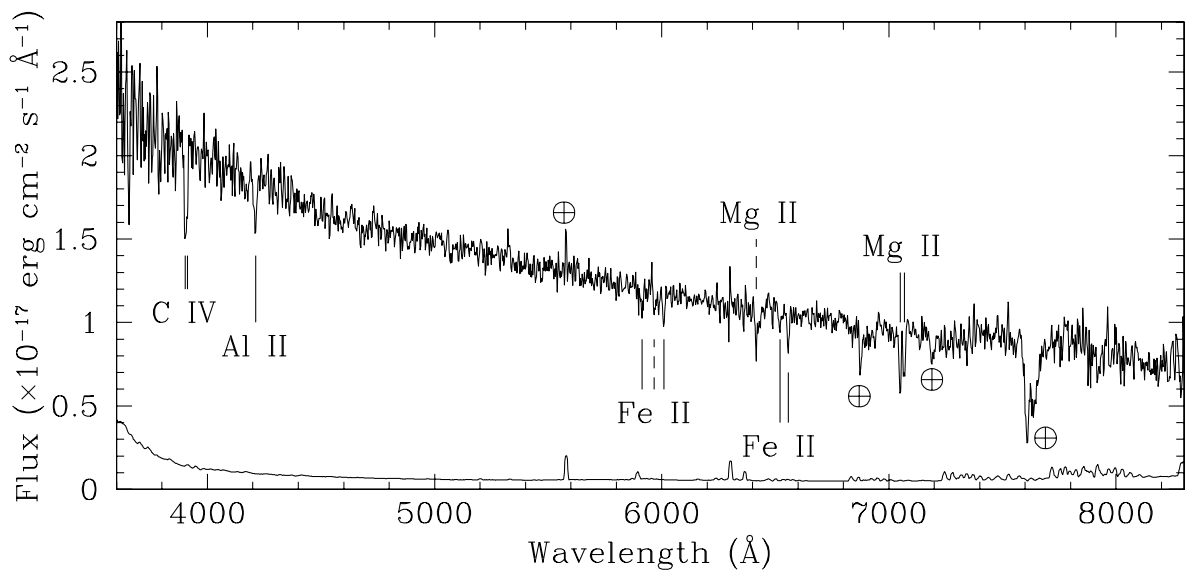

Fig. 3. Optical spectrum of the afterglow of GRB 030328. A number of rest-frame ultraviolet metal absorption lines are detected (see also Table 2). To explain all of them, at least two systems at redshift $z=1.5216$ and $z=1.295$ are required. The system at the highest redshift (the lines of which are indicated with solid hyphens) likely originates in the host galaxy, whereas the lower one is produced by a foreground absorber; the lines of the latter are marked with dashed hyphens. The symbol $\oplus$ indicates atmospheric and telluric features. The solid line towards the bottom of the figure represents the $1 \sigma$ Poisson error spectrum.

For Fe II (see the corresponding Curve of Growth in Fig. 4), we derive $\log N_{\mathrm{Fe} \text { II }}=14.3_{-0.2}^{+0.6}$ and an effective Doppler parameter $b=31_{-10}^{+13} \mathrm{~km} \mathrm{~s}^{-1}$. The column density of $\mathrm{Mg}$ II is not well constrained, but is likely in the range $\log N_{\mathrm{Mg} \text { II }}=13.9-15.3$. For Si II and Al II we derive $\log N_{\mathrm{Al} \mathrm{II}}<16.0$ and $\log N_{\mathrm{Si} \text { II }}<16.0$ assuming a conservative value $b>25 \mathrm{~km} \mathrm{~s}^{-1}$ for the Doppler parameter.

As also suggested from the X-ray spectral fitting (Butler et al. 2005), weak absorption is associated with this GRB environment; thus, we do not expect dust in the neutral gas to obscure significantly the optical afterglow. From the Fe II column density, we estimate $A_{\mathrm{V}}<0.1 \mathrm{mag}$ (Savaglio et al. 2003;
Savaglio \& Fall 2004). The dust extinction in the intervening system at $z=1.295$ is even more negligible.

\subsection{Polarimetry}

In Fig. 5 the polarimetric data analysis results are shown. The intrinsic nature of the OT $V$-band polarization is supported by the relative location of the OT with respect to that of field stars in the $U$ vs. $Q$ plot (Fig. 5, upper panel).

After correcting for spurious field polarization, we found $Q_{\mathrm{OT}}=0.029 \pm 0.008$ and $\mathrm{U}_{\mathrm{OT}}=-0.004 \pm 0.008$. The fit of the data with the relation of Di Serego Alighieri (1997; see Fig. 5, 
Table 2. Identifications, redshifts, oscillator strengths $f_{\lambda}$ and rest-frame equivalent widths $W_{r}$ of absorption lines detected at the host galaxy redshift in the spectrum of the GRB 030328 afterglow, and column densities of the corresponding elements.

\begin{tabular}{lcllc}
\hline \hline Line & Redshift & \multicolumn{1}{c}{$f_{\lambda}$} & $\begin{array}{c}W_{r} \\
(\AA)\end{array}$ & $\begin{array}{c}\log N \\
\left(\mathrm{~cm}^{-2}\right)\end{array}$ \\
\hline C IV $\lambda 1550$ & 1.5225 & 0.0952 & $0.57 \pm 0.17^{a}$ & - \\
C IV $\lambda 1548$ & 1.5206 & 0.1908 & $1.07 \pm 0.20^{a}$ & - \\
Al II $\lambda 1670$ & 1.5211 & 1.833 & $0.84 \pm 0.16$ & $<16.0$ \\
Fe II $\lambda 2600$ & 1.5220 & 0.239 & $0.92 \pm 0.18$ & $14.3_{-0.2}^{+0.6}$ \\
Fe II $\lambda 2586$ & 1.5209 & 0.0691 & $0.47 \pm 0.14$ & $\prime \prime$ \\
Fe II $\lambda 2382$ & 1.5220 & 0.320 & $0.63 \pm 0.15$ & $\prime \prime$ \\
Fe II $\lambda 2374$ & - & 0.0313 & $<0.4$ & $\prime \prime$ \\
Fe II $\lambda 2344$ & 1.5227 & 0.320 & $0.58 \pm 0.14$ & $\prime \prime$ \\
Fe II $\lambda 1608$ & - & 0.058 & $<0.5$ & $\prime \prime$ \\
Mg II $\lambda 2803$ & 1.5211 & 0.3054 & $1.34 \pm 0.19$ & $13.9-15.3$ \\
Mg II $\lambda 2796$ & 1.5212 & 0.6123 & $1.57 \pm 0.20$ & $\prime \prime$ \\
Si II $\lambda 1526$ & - & 0.126 & $<0.6$ & $<16.0$ \\
Al III $\lambda 1854$ & - & 1.833 & $<0.5$ & $<14.3$ \\
\hline
\end{tabular}

${ }^{a}$ The $\mathrm{C}$ IV doublet is blended.

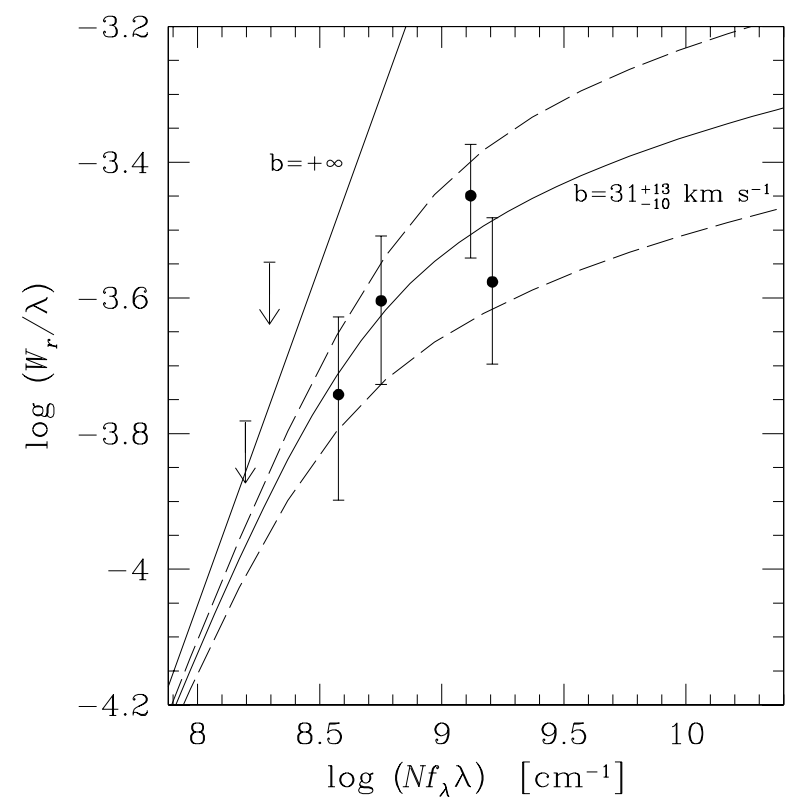

Fig. 4. Curve of Growth for the Fe II absorption lines detected in the GRB 030328 OT spectrum at redshift $z=1.5216$. The curved solid line represent the best fit, whereas the straight solid line marks the $b=$ $+\infty$ case. Dashed lines indicate the 1- $\sigma$ borders of the best-fit curve.

lower panel) yielded for the OT a linear polarization percentage of $P=(2.4 \pm 0.6) \%$ and a polarization angle $\theta=170^{\circ} \pm 7^{\circ}$, corrected for the polarization bias (Wardle \& Kronberg 1974). This latter correction is introduced because $P$ is a positive definite quantity, and thus at low signal-to-noise $(\mathrm{S} / \mathrm{N})$ polarization levels the distribution function of $P$ is no longer normal but becomes skewed, which causes an overestimate of the real value of $P$ (Simmons \& Stewart 1985).

The polarimetric observations covered the time interval 0.66-0.88 d after the GRB, during which the total flux of the OT decreased by $\sim 0.5$ mag assuming the afterglow temporal decay as modeled in Sect. 3.2. In order to check whether this could have affected our measurements, we also separately considered each of the 5 single polarimetry cycles (see Table 3 ). Although with lower $\mathrm{S} / \mathrm{N}, P$ and $\theta$ are consistent with being constant across the whole polarimetric observation run (i.e., over $\sim 5.3 \mathrm{~h}$ ). This
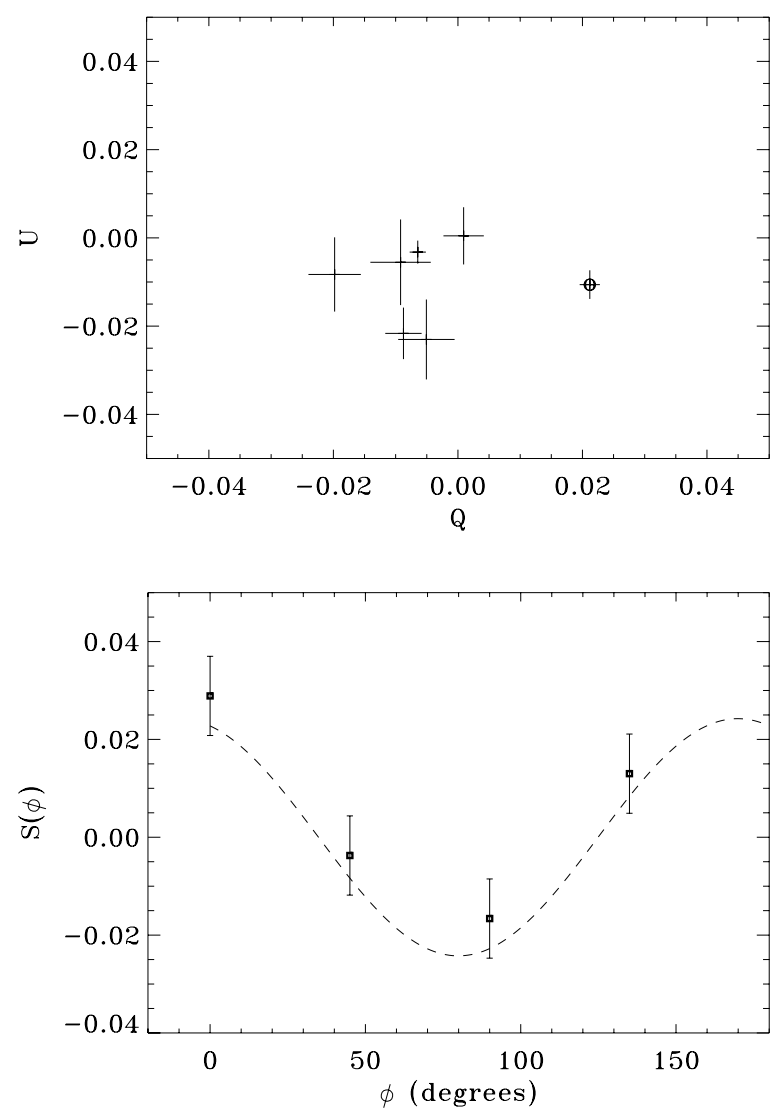

Fig. 5. (Upper panel) Positions of field stars and of the OT (marked with the open circle) in the plane of $Q$ and $U$ parameters not corrected for spurious (instrumental plus field) polarization. The OT is clearly separated from the region occupied by the field stars: this indicates that it has net intrinsic polarization. (Lower panel) Cosine fit of our $V$-band polarimetric data. The best-fit yields $P=(2.4 \pm 0.6) \%$ and $\theta=170^{\circ} \pm 7^{\circ}$.

justifies our choice to consider the total polarimetric images, summed over each angle, for the determination of the $P$ and $\theta$ values in order to increase the final $\mathrm{S} / \mathrm{N}$ of the measurement.

The use of standard polarization equations (e.g., Ramaprakash 1998) leads to results which are in good agreement, within the uncertainties, with those obtained from the method described above.

Once the correction for spurious polarization, induced by the Galactic ISM, has been taken into account, one may be concerned with the presence of further spurious polarization effects local to the GRB host. However, regarding possible additional local dust absorption within the host, we have shown in Sect. 3.3 that we do not find any evidence for this, based on the analysis of the optical spectrum of the GRB 030328 afterglow. Moreover, the contribution of the host galaxy to the total (OT+host) light is just a few percent; therefore we do not expect that the host emission significantly contaminated our polarization measurement. Therefore we conclude that the measured OT polarization is intrinsic.

\subsection{The broadband spectrum of the afterglow}

By using the available information, we have constructed the optical-to-X-ray SFD of the GRB 030328 afterglow at 0.78 days after the GRB, that is, the epoch with best broadband photometric coverage. 
Table 3. $P$ and $\theta$ values measured in each of the 5 cycles of $V$-band polarimetry performed on the GRB 030328 afterglow. $\Delta t$ is the time range of each polarization cycle expressed in days after the GRB trigger.

\begin{tabular}{cccc}
\hline \hline Cycle & $\Delta t$ & $P(\%)$ & $\theta\left(^{\circ}\right)$ \\
\hline 1 & $0.6570-0.6952$ & $2.8 \pm 1.1$ & $172 \pm 11$ \\
2 & $0.7165-0.7546$ & $2.6 \pm 1.1$ & $172 \pm 12$ \\
3 & $0.7564-0.7946$ & $1.8 \pm 1.3$ & $170 \pm 19$ \\
4 & $0.7981-0.8364$ & $2.5 \pm 1.2$ & $177 \pm 14$ \\
5 & $0.8386-0.8768$ & $2.7 \pm 1.5$ & $173 \pm 16$ \\
\hline
\end{tabular}

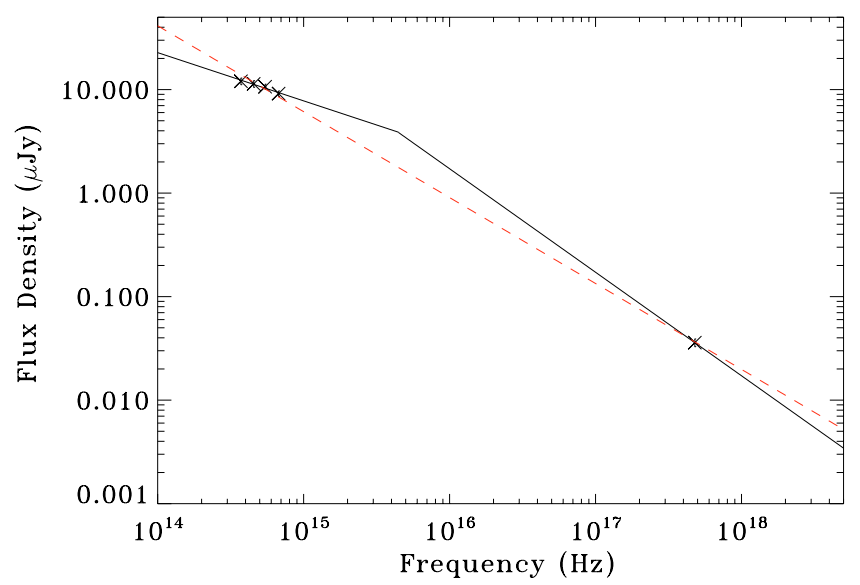

Fig. 6. Broadband spectrum at the epoch corresponding to $0.78 \mathrm{~d}$ after the GRB. The dashed line corresponds to a fit of optical and X-ray bands using a single powerlaw with a spectral index of 0.8 ; the broken solid line indicates that a good fit is also obtained assuming the cooling frequency between optical and X-ray bands, at $5.9 \times 10^{15} \mathrm{~Hz}$.

The optical flux densities at the wavelengths of the $B V R I$ bands have been derived by subtracting the host galaxy contribution at the epoch we selected. As in Sect. 2.1, we assumed $E(B-V)=0.047$ to deredden the data and the normalizations given in Fukugita et al. (1995) to obtain the corresponding flux densities in the optical band. When needed, we rescaled the data to the corresponding reference epoch, 2003 Mar. 29.253 UT, using the optical powerlaw decay with (post-break) index $\alpha_{\text {opt }}=1.50 \pm 0.07$ as the SFD reference epoch is after $t_{\mathrm{b}}$. We also rescaled to the SFD epoch the X-ray flux corresponding to the logarithmic center of the X-ray spectrum energy range (0.5-5 keV, see Butler et al. 2005): this was done using the powerlaw decay found by these authors for the $\mathrm{X}$-ray afterglow temporal behaviour, $\alpha_{\mathrm{X}}=1.5 \pm 0.1$.

As Fig. 6 shows, we fitted the data in two different ways. First we used a single powerlaw (dashed line) to fit the optical and the X-ray data, and we found a spectral index $\beta_{\mathrm{X}-\mathrm{opt}}=0.83 \pm$ 0.01 . However, when we independently fit the optical data with a powerlaw, we obtain a spectral slope $\beta_{\text {opt }}=0.47 \pm 0.15$, which is marginally flatter than the X-ray one $\left(\beta_{X}=\Gamma_{X}-1=1.0 \pm 0.2\right.$; Butler et al. 2005).

In this latter case, represented by the broken solid line in Fig. 6, the presence of a spectral steepening between optical and $\mathrm{X}$-ray bands could be explained with the presence of a cooling frequency $v_{\mathrm{c}}$ in the framework of the synchrotron fireball model (Sari et al. 1998). Assuming a negligible host absorption (see Sect. 3.3) and using the optical and X-ray slopes above, we obtain $v_{\mathrm{c}}=5.9 \times 10^{15} \mathrm{~Hz}$.

\section{Discussion}

The afterglow of GRB 030328 was imaged in the optical for $\sim 2.5$ days, starting a few hours after the explosion. Optical spectroscopy and polarimetry have also been performed. By combining these data with observations at other frequencies, we can compare the temporal and spectral behaviour of its broad-band afterglow with the fireball scenario, assuming a regime of adiabatic expansion (Sari et al. 1998, 1999).

The steepening of the $R$-band light curve around 0.5 days after the GRB suggests the presence of a jet break and makes the scenario of a spherical expansion less probable. Therefore, hereafter we will consider only a jet-collimated expansion in a homogeneous medium (Sari et al. 1999; Rhoads 1999) or in a wind-shaped medium (Chevalier \& Li 2000).

The optical and X-ray spectral indices $\left(\beta_{\mathrm{opt}}=0.47 \pm 0.15\right.$ and $\beta_{\mathrm{X}}=1.0 \pm 0.2$ ) are marginally consistent with each other, therefore we cannot exclude that a single power-law with index $\beta_{\text {opt }}=\beta_{\mathrm{X}}=0.83 \pm 0.01$ is the best approximation of the opticalto-X-ray spectrum (see Fig. 6).

The first case we deal with is that in which the optical and $\mathrm{X}$-ray bands have different spectral slopes. Assuming $\beta_{\mathrm{X}}=p / 2$, we obtain $p=2.0 \pm 0.4$ and $\beta_{\text {opt }}=(p-1) / 2=0.5 \pm 0.2$, which is fully consistent with the value we found from the broadband spectrum (see Sect. 3.5). Moreover, if we consider a fireball model consisting of a fixed-angle jet-collimated expansion in a homogeneous medium (Mészáros \& Rees 1999), we obtain $\alpha_{1}^{\text {model }}=3(p-1) / 4=0.75 \pm 0.3$ and $\alpha_{2}^{\text {model }}=3(p-1) / 4+3 / 4=$ $1.5 \pm 0.3$ before and after the jet break, respectively. These values are consistent (within the errors) with the broken powerlaw decay indices of the $R$-band light curve. Moreover, the computed post-break decay index $\alpha_{2}^{\text {model }}$ is consistent with the X-ray temporal slope as determined by Butler et al. (2005), as the Chandra observation starts after the optical jet break time.

In the second case we consider that the optical-to-X-ray spectrum is produced by the same emission component so that it is described by a single spectral slope, $\beta=0.83$. If the cooling frequency $v_{\mathrm{c}}$ is below the optical band $\left(v_{\mathrm{c}}<v_{\mathrm{opt}}\right)$, one would have $\beta_{\mathrm{X}}=\beta_{\mathrm{opt}}=p / 2$, so we obtain for the electron energy distribution index $p$ the value $1.66 \pm 0.02$ and a temporal decay index $\alpha<1$ (see the relations of Dai \& Cheng 2001 for the cases in which $p<2$ ). The latter is however not consistent with the observed optical and X-ray temporal indices at the SFD time. Otherwise, if $v_{\mathrm{c}}>v_{\mathrm{X}}$, which implies $\beta_{\mathrm{X}}=\beta_{\mathrm{opt}}=(p-1) / 2$, we obtain $p=2.66 \pm 0.02$ : also in this case, the value for $\alpha$ after the jet break time (expected to be numerically equal to $p$ ) is not consistent with our observations. Even assuming a fixed collimation angle, the inconsistency between the model and the observations persists.

Similarly, assuming a fireball expansion in a wind-shaped medium (Chevalier \& Li 2000) returns temporal decay values that are inconsistent with those observed. Thus, this option is not viable as a description of the GRB 030328 afterglow evolution.

We conclude that the favoured scenario is a jet-collimated expansion in a homogeneous medium with fixed opening angle. From the measured jet break time we can compute the jet opening angle value for GRB 030328 which is, following Sari et al. (1999), $\theta_{\text {jet }} \sim 3.2$.

In this hypothesis, considering a luminosity distance $d_{\mathrm{L}}=$ $11.96 \mathrm{Gpc}$ and a GRB fluence $S_{\gamma}=3.0 \times 10^{-5} \mathrm{erg} \mathrm{cm}$ (Villasenor et al. 2003), we find that $E_{\text {iso }}=k \cdot\left(4 \pi S_{\gamma} d_{\mathrm{L}}^{2}\right) /(1+z) \sim$ $2.0 \times 10^{53} \mathrm{erg}$, where $k$ is the the bolometric correction factor, which we assume to be $\sim 1$ (Bloom et al. 2001). With the information above, we can estimate the value of the total energy 
corrected for the jet collimation as $E_{\gamma} \sim 3.1 \times 10^{50} \mathrm{erg}$. This $E_{\gamma}$ value, when compared with the rest-frame peak energy $E_{\text {peak }}$ of the $v F_{v}$ spectrum of the prompt event (Barraud et al. 2004) is consistent within $1 \sigma$ with the "Ghirlanda relation" (Ghirlanda et al. 2004) which ties these two quantities together.

The presence of a bump during the first phases of the optical decay is not uncommon in GRB OTs (see e.g. GRB 000301c, Masetti et al. 2000; GRB 021004, de Ugarte Postigo et al. 2005; GRB 050502a; Guidorzi et al. 2005). This behaviour has generally been interpreted as due to late energy injection produced by a refreshed shock (Kumar \& Piran 2000; Björnsson et al. 2004). This mechanism may explain the early bump we possibly see starting $\sim 0.2 \mathrm{~d}$ after the high-energy prompt event in the OT light curves of GRB 030328, although a detailed analysis is hindered by the low $\mathrm{S} / \mathrm{N}$ of the feature.

Concerning optical spectroscopy, we note that the absorption system at $z=1.5216$, likely associated with the host of GRB 030328 (Fig. 3), is one of the weakest ever observed in a GRB optical transient. The rest-frame EW of the Fe II $\lambda 2600$ line (Table 2) is only $0.9 \AA$ and the total EW of the $\mathrm{Mg}$ II doublet is $2.9 \AA$. To put this in context, all the $\mathrm{Mg}$ II and Fe II $\lambda 2600$ absorptions detected so far in GRB afterglows ( $\sim 10$ objects) are larger than $3 \AA$ and $1 \AA$, respectively, with the exception of GRBs 021004 (Fiore et al. 2005; Mirabal et al. 2003) and 990510 (Vreeswijk et al. 2001). Indeed GRB 990510 , studied by Vreeswijk et al. (2001), has Mg II and Fe II $\lambda 2600$ EWs very similar to those of GRB 030328. Incidentally, the spectral slope of the optical afterglow in these two GRB OTs are also similar: assuming $F_{\lambda} \propto \lambda^{-\delta}$, one finds $\delta \simeq 1.5$ in both cases.

We also note that the GRB 030328 host may be a GRBDamped Lyman- $\alpha$ Absorber (GRB-DLA; Vreeswijk et al. 2004): indeed, in DLA galaxies it was found that Fe II $\lambda 2600$ and $\mathrm{Mg}$ II $\lambda 2796 \mathrm{EWs}$ are larger than $0.5 \AA$, as is seen in the GRB 030328 OT spectrum.

The detection of net polarization may imply that some kind of asymmetry is present in the GRB 030328 afterglow emission. The most natural explanation is the presence of a jet geometry, which is thought to produce the polarization observed in GRB OTs (Sari 1999; Ghisellini \& Lazzati 1999). The fact that we detect optical polarization around the jet break time, and that it does not seem to vary in percentage and angle during our five polarimetric subsets acquired over a time span of $\sim 5.3 \mathrm{~h}$, argues against the possibility that it is produced in a homogeneous jet. Indeed, in this case two polarization peaks are expected, separated by a moment of null polarization (roughly coincident with the break time of the total light curve) over which the polarization angle changes by $90^{\circ}$ (see e.g. Ghisellini \& Lazzati 1999; Lazzati et al. 2003; Rossi et al. 2004). So, we would expect that $P$ vanishes at $t_{\mathrm{b}}$ and that it and $\theta$ rapidly change (on timescales of tens of minutes; see Fig. 5 of Lazzati et al. 2003) across that epoch; however, no hint for both facts is found in the case of the GRB 030328 OT (see Table 3). Rather, the presence of net polarization and the constancy of $P$ and $\theta$ are expected around the jet break time if a structured jet is present (Rossi et al. 2004): thus, we suggest that the collimated emission of GRB 030328 has a structured distribution of the emissivity across the jet aperture.

Acknowledgements. We thank the anonymous referee for several useful comments which helped us to improve the paper. We also thank the ESO staff astronomers for their help and efforts in obtaining the observations presented in this paper, and J. Greiner for useful comments on an earlier version of this manuscript. We are grateful to John Stephen for the language editing of the final version of the manuscript. We acknowledge Scott Barthelmy for maintaining the GRB Coordinates Network (GCN) and BACODINE services. This research has made use of NASA's Astrophysics Data System and HEASARC high-energy missions archive, and of data retrieved from the ESO/ST-ECF Science Archive. This work has been partially supported by the EU RTN Contract HPRN-CT-2002-00294.

\section{References}

Assafin, M., Andrei, A. H., Vieira Martins, R., et al. 2001, ApJ, 552, 380 Barraud, C., Atteia, J. L., Olive, J.-F., et al. 2004, Spectral analysis of 50 GRBs detected by HETE-2, in Gamma-Ray Bursts: 30 Years of Discovery: Gamma-Ray Burst Symposium, ed. E. E. Fenimore, \& M. Galassi (Melville, NY: American Institute of Physics), AIP Conf. Proc., 727, 81

Bartolini, C., Guarnieri, A., Piccioni, A., Gualandi, R., \& Pizzichini, G. 2003, GCN Circ., 2008

Beuermann, K., Hessman, F. V., Reinsch, K., et al. 1999, A\&A, 352, L26 Björnsson, G., Gudmundsson, E. H., \& Jóhannesson, G. 2004, ApJ, 615, L77 Bloom, J. S., Frail, D. A., \& Sari, R. 2001, AJ, 121, 2879

Burenin, R., Denissenko, D., Pavlinsky, M., et al. 2003, GCN Circ., 1990

Butler, N. R., Ricker, G. R., Ford, P. G., et al. 2005, ApJ, 629, 908 Cardelli, J. A., Clayton, G. C., \& Mathis, J. S. 1989, ApJ, 345, 245

Chevalier, R. A., \& Li, Z. Y. 2000, ApJ, 536, 195

Costa, E., Frontera, F., Heise, J., et al. 1997, Nature, 387, 783

Covino, S., Lazzati, D., Ghisellini, G., et al. 1999, 348, L1

Dai, Z. G., \& Cheng, K. S. 2001, ApJ, 558, L109

de Ugarte Postigo, A., Castro-Tirado, A. J., Gorosabel, J., et al. 2005, A\&A, 443, 841

Deutsch, E. W. 1999, AJ, 118, 1882

Di Serego Alighieri, S. 1997, Polarimetry with large telescopes, in Instrumentation for large telescopes, ed. J. M. Rodriguez Espinosa, A. Herrero, \& F. Sánchez (Cambridge: Cambridge Univ. Press), 287

Dickey, J. M., \& Lockman, F. J. 1990, ARA\&A, 28, 215

Fiore, F., D’Elia, V., Lazzati, D., et al. 2005, ApJ, 624, 853

Frail, D. A., Kulkarni, S. R., Nicastro, L., Feroci, M., \& Taylor, G. B. 1997, Nature, 389, 261

Fugazza, D., Fiore, F., Cocchia, M., et al. 2003a, GCN Circ., 1983

Fugazza, D., Antonelli, L. A., Fiore, F., et al. 2003b, GCN Circ., 1982

Fukugita, M., Shimasaku, K., \& Ichikawa, T. 1995, PASP, 107, 945

Galama, T. J., Wijers, R. A. M. J., Bremer, M., et al. 1998a, ApJ, 500, L97

Galama, T. J., Vreeswijk, P. M., van Paradijs, J., et al. 1998b, Nature, 395, 670

Gal-Yam, A., Ofek, E. O., \& Polishook, D. 2003, GCN Circ., 1984

Garnavich, P., Martini, P., \& Stanek, K. Z. 2003, GCN Circ., 2036

Ghirlanda, G., Ghisellini, G., \& Lazzati, D. 2004, ApJ, 616, 331

Ghisellini, G., \& Lazzati, D. 1999, MNRAS, 309, L7

Gorosabel, J., Jelínek, M., de Ugarte Postigo, A., et al. 2005, The GRB 030328 host: Another case of a blue starburst galaxy, in 4th Workshop - GammaRay Burst in the Afterglow Era, ed. L. Piro, L. Amati, S. Covino, \& B. Gendre, Il Nuovo Cimento, 28C, 677

Greiner, J., Klose, S., Reinsch, K., et al. 2003a, Nature, 426, 157

Greiner, J., Peimbert, M., Estaban, C., et al. 2003b, GCN Circ., 2020

Guarnieri, A., Bartolini, C., Masetti, N., et al. 1997, A\&A, 328, L13

Guidorzi, C., Monfardini, A., Gomboc, A., et al. 2005, ApJ, 630, L121

Hamuy, M., Walker, A. R., Suntzeff, N. B., et al. 1992, PASP, 104, 533

Hamuy, M., Suntzeff, N. B., Heathcote, S. R., et al. 1994, PASP, 106, 566

Henden, A. A. 2003, GCN Circ., 2114

Hjorth, J., Sollerman, J., Møller, P., et al. 2003, Nature, 423, 847

Ibrahimov, M. A., Asfandiyarov, I. M., Kahharov, B. B., et al. 2003, GCN Circ., 2192

Iwamoto, K., Mazzali, P. A., Nomoto, K., et al. 1998, Nature, 395, 672

Kouveliotou, C., Meegan, C. A., Fishman, G. J., et al. 1993, ApJ, 413, L101

Kumar, P., \& Piran, T. 2000, ApJ, 532, 286

Lazzati, D., Covino, S., Di Serego Alighieri, S., et al. 2003, A\&A, 410, 823

Malesani, D., Tagliaferri, G., Chincarini, G., et al. 2004, ApJ, 609, L5

Martini, P., Garnavich, P., \& Stanek, K. Z. 2003a, GCN Circ., 1980

Martini, P., Garnavich, P., \& Stanek, K. Z. 2003b, GCN Circ., 1979

Masetti, N., Pian, E., Palazzi, E., et al. 1999, A\&AS, 138, 453

Masetti, N., Bartolini, C., Bernabei, S., et al. 2000, A\&A, 359, L23

Matheson, T., Garnavich, P. M., Stanek, K. Z., et al. 2003, ApJ, 599, 394

Mészáros, P., \& Rees, M. J. 1999, MNRAS, 306, L39

Mirabal, N., Halpern, J. P., Chornock, R., et al. 2003, ApJ, 595, 935

Palazzi, E., Pian, E., Masetti, N., et al. 1998, A\&A, 336, L95

Peterson, B. A., \& Price, P. A. 2003, GCN Circ., 1974

Price, P. A., \& Peterson, B. A. 2003, GCN Circ., 1977

Ramaprakash, A. N. 1998, Ph.D. Thesis, Inter-University Centre for Astronomy and Astrophysics (http://www .iucaa.ernet.in/ anr/thesis.html) 
Rhoads, J. E. 1999, ApJ, 525, 737

Rol, E., Wijers, R. A. M. J., Vreeswijk, P. M., et al. 2000, ApJ, 544, 707 Rol, E., Wijers, R. A. M. J., Fynbo, J. P. U., et al. 2003a, A\&A, 405, L23 Rol, E., Vreeswijk, P., \& Jaunsen, A. 2003b, GCN Circ., 1981

Rossi, E. M., Lazzati, D., Salmonson, J. D., \& Ghisellini, G. 2004, MNRAS, 354,86

Rumyantsev, V., Biryukov, V., \& Pozanenko, A. 2003, GCN Circ., 1991

Sari, R. 1999, ApJ, 524, L43

Sari, R., Piran, T., \& Narayan, R. 1998, ApJ, 497, L17

Sari, R., Piran, T., \& Halpern, J. P. 1999, ApJ, 519, L17

Savaglio, S., \& Fall, S. M. 2004, ApJ, 614, 293

Savaglio, S., Fall, S. M., \& Fiore, F. 2003, ApJ, 585, 638

Schlegel, D. J., Finkbeiner, D. P., \& Davis, M. 1998, ApJ, 500, 525
Simmons, J. F. L. \& Stewart, B. G. 1985, A\&A, 142, 100

Šimon, V., Hudec, R., Pizzichini, G., \& Masetti, N. 2001, A\&A, 377, 450 Stanek, K. Z., Matheson, T., Garnavich, P. M., et al. 2003, ApJ, 591, L17 Stetson, P. B. 1987, PASP, 99, 191

Vanderspek, R., Crew, G., Doty, J., et al. 2003, GCN Circ., 1997 van Dokkum, P. G. 2001, PASP, 113, 1420

van Paradijs, J., Groot, P. J., Galama, T. J., et al. 1997, Nature, 386, 686 Villasenor, J., Crew, G., Vanderspek, R., et al. 2003, GCN Circ., 1978

Vreeswijk, P. M., Fruchter, A., Kaper, L., et al. 2001, ApJ, 546, 672

Vreeswijk, P. M., Ellison, S., Ledoux, C., et al. 2004, A\&A, 419, 927 Wardle, J. F. C., \& Kronberg, P. P. 1974, ApJ, 194, 249

Wijers, R. A. M. J., Vreeswijk, P. M., Galama, T. J., et al. 1999, ApJ, 523, L33 Woosley, S. E., \& Heger, A. 2006, ApJ, 637, 914 
E. Maiorano et al.: Physics of the GRB 030328 afterglow and its environment, Online Material p 1

\section{Online Material}


E. Maiorano et al.: Physics of the GRB 030328 afterglow and its environment, Online Material $p 2$

Table 1. Log of the photometric, spectroscopic and polarimetric observations presented in this paper. No corrections for foreground Galactic absorption and for the underlying host contribution were applied to the reported magnitudes. Magnitude errors are at $1 \sigma$ confidence level, whereas upper limits are at $3 \sigma$ confidence level.

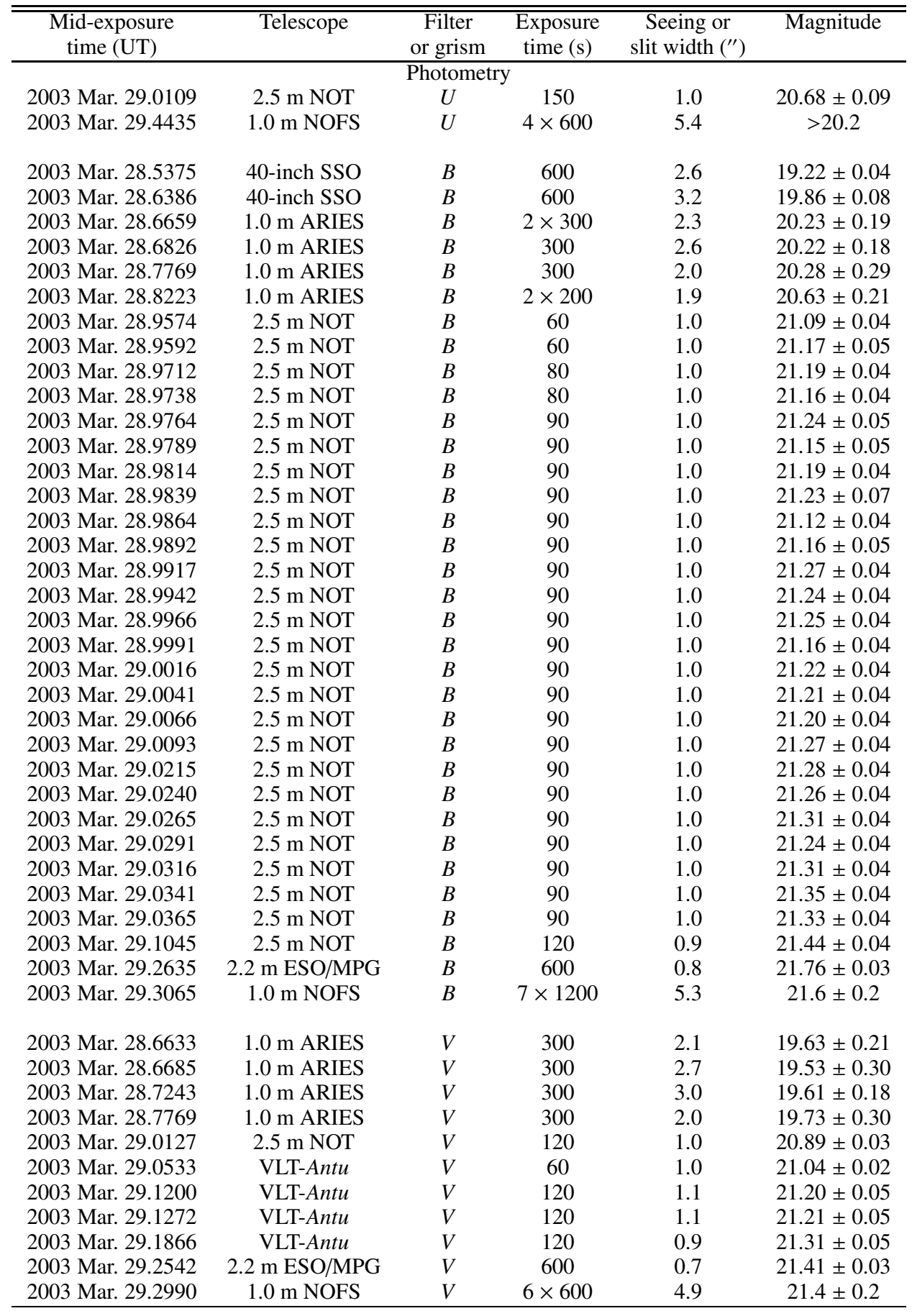


Table 1. continued.

\begin{tabular}{|c|c|c|c|c|c|}
\hline $\begin{array}{l}\text { Mid-exposure } \\
\text { time (UT) }\end{array}$ & Telescope & $\begin{array}{c}\begin{array}{c}\text { Filter } \\
\text { or grism }\end{array} \\
\end{array}$ & $\begin{array}{l}\text { Exposure } \\
\text { time }(\mathrm{s})\end{array}$ & $\begin{array}{c}\text { Seeing or } \\
\text { slit width (') }\end{array}$ & Magnitude \\
\hline \multicolumn{6}{|c|}{ Photometry } \\
\hline 2003 Mar. 28.5296 & 40-inch SSO & $R$ & 600 & 2.2 & $18.59 \pm 0.04$ \\
\hline 2003 Mar. 28.5463 & 40-inch SSO & $R$ & 600 & 2.6 & $18.74 \pm 0.03$ \\
\hline 2003 Mar. 28.5542 & 40-inch SSO & $R$ & 600 & 2.4 & $18.81 \pm 0.03$ \\
\hline 2003 Mar. 28.6305 & 40-inch SSO & $R$ & 600 & 2.6 & $19.33 \pm 0.05$ \\
\hline 2003 Mar. 28.6441 & $1.0 \mathrm{~m}$ ARIES & $R$ & $200+300$ & 2.7 & $19.45 \pm 0.11$ \\
\hline 2003 Mar. 28.6917 & $1.0 \mathrm{~m}$ ARIES & $R$ & 300 & 1.9 & $19.53 \pm 0.14$ \\
\hline 2003 Mar. 28.7570 & $1.0 \mathrm{~m}$ ARIES & $R$ & 300 & 2.0 & $19.55 \pm 0.18$ \\
\hline 2003 Mar. 28.7991 & $1.0 \mathrm{~m}$ ARIES & $R$ & 300 & 1.7 & $19.84 \pm 0.14$ \\
\hline 2003 Mar. 28.9447 & $2.5 \mathrm{~m}$ NOT & $R$ & 20 & 1.0 & $20.51 \pm 0.08$ \\
\hline 2003 Mar. 28.9462 & $2.5 \mathrm{~m}$ NOT & $R$ & 20 & 1.0 & $20.43 \pm 0.07$ \\
\hline 2003 Mar. 28.9484 & $2.5 \mathrm{~m} \mathrm{NOT}$ & $R$ & 60 & 1.0 & $20.50 \pm 0.04$ \\
\hline 2003 Mar. 28.9525 & $2.5 \mathrm{~m} \mathrm{NOT}$ & $R$ & 60 & 1.0 & $20.45 \pm 0.03$ \\
\hline 2003 Mar. 28.9540 & $2.5 \mathrm{~m}$ NOT & $R$ & 60 & 1.0 & $20.46 \pm 0.04$ \\
\hline 2003 Mar. 28.9548 & $2.5 \mathrm{~m} \mathrm{NOT}$ & $R$ & 60 & 1.0 & $20.44 \pm 0.04$ \\
\hline 2003 Mar. 28.9556 & $2.5 \mathrm{~m} \mathrm{NOT}$ & $R$ & 30 & 1.0 & $20.41 \pm 0.05$ \\
\hline 2003 Mar. 28.9562 & $2.5 \mathrm{~m}$ NOT & $R$ & 30 & 1.0 & $20.52 \pm 0.05$ \\
\hline 2003 Mar. 28.9567 & $2.5 \mathrm{~m} \mathrm{NOT}$ & $R$ & 30 & 1.0 & $20.55 \pm 0.05$ \\
\hline 2003 Mar. 28.9584 & $2.5 \mathrm{~m} \mathrm{NOT}$ & $R$ & 30 & 1.0 & $20.51 \pm 0.05$ \\
\hline 2003 Mar. 28.9602 & $2.5 \mathrm{~m} \mathrm{NOT}$ & $R$ & 40 & 1.0 & $20.46 \pm 0.04$ \\
\hline 2003 Mar. 28.9607 & $2.5 \mathrm{~m} \mathrm{NOT}$ & $R$ & 40 & 1.0 & $20.49 \pm 0.05$ \\
\hline 2003 Mar. 28.9613 & $2.5 \mathrm{~m} \mathrm{NOT}$ & $R$ & 40 & 1.0 & $20.44 \pm 0.05$ \\
\hline 2003 Mar. 28.9618 & $2.5 \mathrm{~m} \mathrm{NOT}$ & $R$ & 40 & 1.0 & $20.49 \pm 0.05$ \\
\hline 2003 Mar. 28.9624 & $2.5 \mathrm{~m}$ NOT & $R$ & 40 & 1.0 & $20.46 \pm 0.05$ \\
\hline 2003 Mar. 28.9630 & $2.5 \mathrm{~m}$ NOT & $R$ & 40 & 1.0 & $20.50 \pm 0.05$ \\
\hline 2003 Mar. 28.9635 & $2.5 \mathrm{~m} \mathrm{NOT}$ & $R$ & 40 & 1.0 & $20.41 \pm 0.08$ \\
\hline 2003 Mar. 28.9641 & $2.5 \mathrm{~m} \mathrm{NOT}$ & $R$ & 40 & 1.0 & $20.39 \pm 0.08$ \\
\hline 2003 Mar. 28.9647 & $2.5 \mathrm{~m} \mathrm{NOT}$ & $R$ & 40 & 1.0 & $20.49 \pm 0.09$ \\
\hline 2003 Mar. 28.9652 & $2.5 \mathrm{~m} \mathrm{NOT}$ & $R$ & 40 & 1.0 & $20.47 \pm 0.07$ \\
\hline 2003 Mar. 28.9658 & $2.5 \mathrm{~m}$ NOT & $R$ & 40 & 1.0 & $20.54 \pm 0.07$ \\
\hline 2003 Mar. 28.9663 & $2.5 \mathrm{~m}$ NOT & $R$ & 40 & 1.0 & $20.51 \pm 0.07$ \\
\hline 2003 Mar. 28.9669 & $2.5 \mathrm{~m} \mathrm{NOT}$ & $R$ & 40 & 1.0 & $20.50 \pm 0.08$ \\
\hline 2003 Mar. 28.9675 & $2.5 \mathrm{~m} \mathrm{NOT}$ & $R$ & 40 & 1.0 & $20.49 \pm 0.07$ \\
\hline 2003 Mar. 28.9680 & $2.5 \mathrm{~m} \mathrm{NOT}$ & $R$ & 40 & 1.0 & $20.46 \pm 0.05$ \\
\hline 2003 Mar. 28.9689 & $2.5 \mathrm{~m} \mathrm{NOT}$ & $R$ & 60 & 1.0 & $20.53 \pm 0.04$ \\
\hline 2003 Mar. 28.9700 & $2.5 \mathrm{~m} \mathrm{NOT}$ & $R$ & 80 & 1.0 & $20.52 \pm 0.03$ \\
\hline 2003 Mar. 28.9725 & $2.5 \mathrm{~m}$ NOT & $R$ & 80 & 1.0 & $20.51 \pm 0.04$ \\
\hline 2003 Mar. 28.9751 & $2.5 \mathrm{~m}$ NOT & $R$ & 90 & 1.0 & $20.49 \pm 0.03$ \\
\hline 2003 Mar. 28.9777 & $2.5 \mathrm{~m} \mathrm{NOT}$ & $R$ & 90 & 1.0 & $20.52 \pm 0.04$ \\
\hline 2003 Mar. 28.9801 & $2.5 \mathrm{~m} \mathrm{NOT}$ & $R$ & 90 & 1.0 & $20.49 \pm 0.05$ \\
\hline 2003 Mar. 28.9827 & $2.5 \mathrm{~m} \mathrm{NOT}$ & $R$ & 90 & 1.0 & $20.59 \pm 0.05$ \\
\hline 2003 Mar. 28.9852 & $2.5 \mathrm{~m} \mathrm{NOT}$ & $R$ & 90 & 1.0 & $20.54 \pm 0.03$ \\
\hline 2003 Mar. 28.9877 & $2.5 \mathrm{~m} \mathrm{NOT}$ & $R$ & 90 & 1.0 & $20.53 \pm 0.05$ \\
\hline 2003 Mar. 28.9905 & $2.5 \mathrm{~m}$ NOT & $R$ & 90 & 1.0 & $20.54 \pm 0.03$ \\
\hline 2003 Mar. 28.9929 & $2.5 \mathrm{~m}$ NOT & $R$ & 90 & 1.0 & $20.56 \pm 0.03$ \\
\hline 2003 Mar. 28.9954 & $2.5 \mathrm{~m} \mathrm{NOT}$ & $R$ & 90 & 1.0 & $20.59 \pm 0.03$ \\
\hline 2003 Mar. 28.9979 & $2.5 \mathrm{~m} \mathrm{NOT}$ & $R$ & 90 & 1.0 & $20.61 \pm 0.03$ \\
\hline 2003 Mar. 29.0004 & $2.5 \mathrm{~m} \mathrm{NOT}$ & $R$ & 90 & 1.0 & $20.59 \pm 0.03$ \\
\hline 2003 Mar. 29.0029 & $2.5 \mathrm{~m} \mathrm{NOT}$ & $R$ & 90 & 1.0 & $20.61 \pm 0.03$ \\
\hline 2003 Mar. 29.0054 & $2.5 \mathrm{~m} \mathrm{NOT}$ & $R$ & 90 & 1.0 & $20.61 \pm 0.03$ \\
\hline 2003 Mar. 29.0080 & $2.5 \mathrm{~m}$ NOT & $R$ & 90 & 1.0 & $20.65 \pm 0.03$ \\
\hline 2003 Mar. 29.0177 & $2.5 \mathrm{~m}$ NOT & $R$ & 90 & 1.0 & $20.69 \pm 0.03$ \\
\hline 2003 Mar. 29.0202 & $2.5 \mathrm{~m}$ NOT & $R$ & 90 & 1.0 & $20.66 \pm 0.03$ \\
\hline 2003 Mar. 29.0228 & $2.5 \mathrm{~m} \mathrm{NOT}$ & $R$ & 90 & 1.0 & $20.63 \pm 0.03$ \\
\hline 2003 Mar. 29.0252 & $2.5 \mathrm{~m} \mathrm{NOT}$ & $R$ & 90 & 1.0 & $20.64 \pm 0.03$ \\
\hline 2003 Mar. 29.0278 & $2.5 \mathrm{~m} \mathrm{NOT}$ & $R$ & 90 & 1.0 & $20.64 \pm 0.03$ \\
\hline 2003 Mar. 29.0303 & $2.5 \mathrm{~m} \mathrm{NOT}$ & $R$ & 90 & 1.0 & $20.70 \pm 0.03$ \\
\hline 2003 Mar. 29.0328 & $2.5 \mathrm{~m}$ NOT & $R$ & 90 & 1.0 & $20.68 \pm 0.03$ \\
\hline 2003 Mar. 29.0353 & $2.5 \mathrm{~m}$ NOT & $R$ & 90 & 1.0 & $20.67 \pm 0.03$ \\
\hline 2003 Mar. 29.0382 & $2.5 \mathrm{~m}$ NOT & $R$ & 90 & 1.0 & $20.72 \pm 0.03$ \\
\hline
\end{tabular}


E. Maiorano et al.: Physics of the GRB 030328 afterglow and its environment, Online Material p 4

Table 1. continued.

\begin{tabular}{|c|c|c|c|c|c|}
\hline $\begin{array}{l}\text { Mid-exposure } \\
\text { time (UT) }\end{array}$ & Telescope & $\begin{array}{c}\text { Filter } \\
\text { or grism }\end{array}$ & $\begin{array}{l}\text { Exposure } \\
\text { time }(\mathrm{s})\end{array}$ & $\begin{array}{c}\text { Seeing or } \\
\text { slit width (") }\end{array}$ & Magnitude \\
\hline \multicolumn{6}{|c|}{ Photometry } \\
\hline 2003 Mar. 29.1020 & $2.5 \mathrm{~m}$ NOT & $R$ & 120 & 1.0 & $20.79 \pm 0.03$ \\
\hline 2003 Mar. 29.1243 & $1.54 \mathrm{~m}$ Danish & $R$ & 600 & 1.0 & $20.91 \pm 0.09$ \\
\hline 2003 Mar. 29.1333 & $1.54 \mathrm{~m}$ Danish & $R$ & 600 & 1.0 & $20.73 \pm 0.07$ \\
\hline 2003 Mar. 29.1486 & $1.54 \mathrm{~m}$ Danish & $R$ & 600 & 1.0 & $21.00 \pm 0.09$ \\
\hline 2003 Mar. 29.1625 & $1.54 \mathrm{~m}$ Danish & $R$ & 600 & 1.0 & $20.85 \pm 0.06$ \\
\hline 2003 Mar. 29.1643 & $2.5 \mathrm{~m} \mathrm{NOT}$ & $R$ & 200 & 1.0 & $20.88 \pm 0.05$ \\
\hline 2003 Mar. 29.1771 & $1.54 \mathrm{~m}$ Danish & $R$ & 600 & 1.0 & $21.00 \pm 0.07$ \\
\hline 2003 Mar. 29.1958 & $1.54 \mathrm{~m}$ Danish & $R$ & 600 & 1.0 & $20.95 \pm 0.07$ \\
\hline 2003 Mar. 29.2229 & $1.54 \mathrm{~m}$ Danish & $R$ & 600 & 1.0 & $21.10 \pm 0.07$ \\
\hline 2003 Mar. 29.2451 & $1.54 \mathrm{~m}$ Danish & $R$ & 600 & 1.0 & $21.03 \pm 0.07$ \\
\hline 2003 Mar. 29.2727 & $2.2 \mathrm{~m} \mathrm{ESO} / \mathrm{MPG}$ & $R$ & 600 & 0.6 & $21.17 \pm 0.04$ \\
\hline 2003 Mar. 29.3035 & $1.54 \mathrm{~m}$ Danish & $R$ & 600 & 1.0 & $21.23 \pm 0.10$ \\
\hline 2003 Mar. 29.3065 & $1.0 \mathrm{~m}$ NOFS & $R$ & $6 \times 600$ & 4.8 & $21.2 \pm 0.2$ \\
\hline 2003 Mar. 29.3236 & $1.54 \mathrm{~m}$ Danish & $R$ & 600 & 1.0 & $21.36 \pm 0.11$ \\
\hline 2003 Mar. 31.1056 & $2.5 \mathrm{~m}$ NOT & $R$ & $2 \times 600$ & 1.4 & $22.64 \pm 0.09$ \\
\hline 2003 Mar. 28.6525 & $1.0 \mathrm{~m}$ ARIES & $I$ & $2 \times 200$ & 2.1 & $18.88 \pm 0.13$ \\
\hline 2003 Mar. 28.6804 & $1.0 \mathrm{~m}$ ARIES & $I$ & 300 & 2.0 & $18.79 \pm 0.29$ \\
\hline 2003 Mar. 28.7339 & $1.0 \mathrm{~m}$ ARIES & $I$ & 300 & 1.9 & $19.25 \pm 0.17$ \\
\hline 2003 Mar. 28.7459 & $1.0 \mathrm{~m}$ ARIES & $I$ & 300 & 1.9 & $19.13 \pm 0.17$ \\
\hline 2003 Mar. 28.7877 & $1.0 \mathrm{~m}$ ARIES & $I$ & 300 & 1.6 & $19.31 \pm 0.22$ \\
\hline 2003 Mar. 28.8318 & $1.0 \mathrm{~m}$ ARIES & $I$ & 300 & 1.6 & $19.41 \pm 0.44$ \\
\hline 2003 Mar. 29.2438 & $2.2 \mathrm{~m} \mathrm{ESO} / \mathrm{MPG}$ & $I$ & 600 & 0.6 & $20.75 \pm 0.05$ \\
\hline 2003 Mar. 29.3140 & $1.0 \mathrm{~m}$ NOFS & $I$ & $6 \times 600$ & 4.9 & $>20.5$ \\
\hline 2003 Mar. 29.0144 & $2.5 \mathrm{~m} \mathrm{NOT}$ & $i$ & 120 & 1.0 & $20.25 \pm 0.05^{*}$ \\
\hline \multicolumn{6}{|c|}{ Spectroscopy } \\
\hline 2003 Mar. 29.0838 & VLT-Antu & $300 \mathrm{~V}$ & $6 \times 600$ & 1.0 & - \\
\hline \multicolumn{6}{|c|}{ Polarimetry } \\
\hline 2003 Mar. 29.2397 & VLT-Antu & V & $5 \times 4 \times 780$ & 1.0 & - \\
\hline
\end{tabular}

*: Calibrated using $I$-band secondary standards (see text). 